Oper Orthop Traumatol 2016 $28: 1$

DOI 10.1007/s00064-015-0437-1

(c) Springer-Verlag

Berlin Heidelberg 2016
Schriftleitung Operative Orthopädie und Traumatologie

\section{Die Zeitschrift Operative Orthopädie und Traumatologie dankt den Gutachtern 2015}

Die Schriftleitung der Zeitschrift Operative Orthopädie und Traumatologie dankt den Herausgebern der Zeitschrift sowie allen weiteren Experten für ihre Unterstützung als wissenschaftliche Gutachter im Jahr 2015. Durch ihren Einsatz garantieren sie im Rahmen des Peer-Review-Verfahrens der eingereichten Manuskripte die hohe Qualität und klinische Relevanz der Zeitschrift Operative Orthopädie und Traumatologie.
Dr. Karlheinz Kalb, Bad Neustadt / Saale Prof. Dr. Michael Klinger, Göttingen PD Dr. Dietmar Krappinger, Innsbruck Dr. Hans-Peter Kundert, Zürich Prof. Dr. Djordje Lazovic, Oldenburg Dr. Steffen Löw, Bad Mergentheim Dr. Christian-Dominik Peterlein, Marburg Dr. Thomas Pillukat, Bad Neustadt / Saale Prof. Dr. Tim Pohlemann, Homburg Dr. Stephan Reppenhagen, Würzburg Dr. Jörg R. Rether, Tübingen

Dr. Martin Richter, Bonn

Dr. Daniel Rikli, Basel

Prof. Dr. Peter Schandelmaier, Deggendorf
Dr. Sven Scheffler, Berlin Prof. Dr. Michael Schädel-Höpfner, Neuss Dr. Alexander Scola, Ulm Dr. Christian Spies, Bad Rappenau Dr. Hartmut Stinus, Northeim Dr. Ulrich Stöckle, Tübingen Dr. Mark Tauber, München PD Dr. Urs von Deimling, Sankt Augustin Dr. Andreas Wagner, Eisenberg PD Dr. Tim Walde, Göttingen Dr. Markus Wambacher, Innsbruck Prof. Dr. Paul Werker, Groningen Prof. Dr. Johannes Zeichen, Minden 